\title{
Effect of Lidocaine and Epinephrine on Human Erythrocyte Shape and Vesiculability of Blood Cells
}

\author{
Tanja Slokar, ${ }^{1}$ Carlos Lopez-Mariscal, ${ }^{2,3}$ Judita Lea Krek, $^{2}$ \\ Roman Štukelj, ${ }^{2}$ Oskar Zupanc, ${ }^{1}$ and Veronika Kralj-Iglič ${ }^{2}$ \\ ${ }^{1}$ Department of Orthopaedic Surgery, Ljubljana University Medical Centre, Zaloška 9, SI-1000 Ljubljana, Slovenia \\ ${ }^{2}$ Laboratory of Clinical Biophysics, Faculty of Health Sciences, University of Ljubljana, Zdravstvena 5, SI-1000 Ljubljana, Slovenia \\ ${ }^{3}$ US Naval Research Laboratory, 4555 Overlook Avenue SW, Washington, DC 20375, USA \\ Correspondence should be addressed to Veronika Kralj-Iglič́; veronika.kralj-iglic@fe.uni-lj.si
}

Received 29 October 2014; Revised 27 December 2014; Accepted 12 January 2015

Academic Editor: Leonid Pryadko

Copyright (C) 2015 Tanja Slokar et al. This is an open access article distributed under the Creative Commons Attribution License, which permits unrestricted use, distribution, and reproduction in any medium, provided the original work is properly cited.

\begin{abstract}
The effect of local anesthetic composed of lidocaine and epinephrine on vesiculability of blood cells and erythrocyte shape was studied. Whole blood and plasma were incubated with lidocaine/epinephrine. Extracellular vesicles were isolated by centrifugation and washing and counted by flow cytometry. Lidocaine/epinephrine and each component alone were added to diluted blood. Shape changes were recorded by micrographs. An ensemble of captured frames was analyzed for populations of discocytes, echinocytes, and stomatocytes by using statistical methods. Incubation of whole blood and blood plasma with lidocaine/epinephrine considerably increased concentration of extracellular vesicles in isolates (for an average factor 3.4 in blood and 2.8 in plasma). Lidocaine/epinephrine caused change of erythrocyte shape from mainly discocytic to mainly stomatocytic (higher than 50\%). Lidocaine alone had even stronger stomatocytic effect (the percent of stomatocytes was higher than 95\%) while epinephrine had echinocytic effect (the percent of echinocytes was higher than $80 \%$ ). The differences were highly statistically significant $\left(p<10^{-8}\right)$ with statistical power $P=1$. Lidocaine/epinephrine induced regions of highly anisotropically curved regions indicating that lidocaine and epinephrine interact with erythrocyte membrane. It was concluded that lidocaine/epinephrine interacts with cell membranes and increases vesiculability of blood cells in vitro.
\end{abstract}

\section{Introduction}

Biological membranes as a condensed matter present a challenge due to complexity of derived interactions (e.g., interactions mediated by curvature and electric field). The features driven by these interactions [1] are highly relevant for cells and organisms; therefore studies of the membrane properties are important in assessment of the effect of various added substances on cells, tissues, and organisms.

Nanostructures of biological membranes have become a subject of increased interest. Of these, extracellular vesicles (EVs) are membrane-enclosed cell fragments which are shed by cells of all types into extracellular solution $[2,3]$. They are more or less mobile in the surrounding fluid and are able to interact with membranes of other cells. Through these interactions, long-distance intercellular transfer of matter and information takes place [4-6]. EVs are being considered for drug delivery [7] and tissue regeneration [8]. Their assessment could be used in prevention of thromboembolic events [9]. EVs harvested from tissues and body fluids could be used as a basis of new materials and technologies that are expected to be superior to others in personalized nanomedicine. However, knowledge on the mechanisms of EV formation, properties, and effects is yet rudimentary.

Mechanisms of budding and nanovesiculation of cell membranes [10-13] are being studied in many fields of medicine and biology [14-23]. In particular, concentration of EVs in blood isolates is a possible indicator of the size of the catalytic surface for blood clot reaction cascade and underlies blood clot formation in blood vessels; EVs represent a major source of tissue factor in peripheral blood while plateletderived EVs affect the formation of thrombin, which is involved in blood clotting [24-26]. The concentration of EVs was found increased in isolates from blood of patients that 
were subject to thromboembolic events of different origins $[27,28]$ including surgery under anesthesia $[29,30]$.

Erythrocytes are a convenient system for study of mechanisms and interactions relevant for cell membrane and have therefore been extensively studied [13, 31-38]. Namely, erythrocytes lack cytoskeleton (aside from the membrane skeleton) so that these mechanisms and interactions affect the cell shape, which can be directly observed by optical microscope.

To better understand the mechanisms of budding and vesiculation of biological membranes, in this work, we studied in vitro effects of a local anesthetic lidocaine/epinephrine (L/E) on blood cell membrane nanovesiculation and erythrocyte shape change.

\section{Materials and Methods}

2.1. Chemicals. Local anesthetic lidocaine/epinephrine (L/E) contained 2\% lidocaine mixed with epinephrine 1:200000 (Xylanaest, Gebro Pharma GmbH). Lidocaine (L) used in in vitro experiments with blood cells contained $20 \mathrm{mg}$ Lidocaini hydrochloridum (SIMS, Florence, Italy), $4.8 \mathrm{mg}$ sodium chloride (Merck, Darmstadt, Germany), water, and $\mathrm{NaOH}$ for adjustment of $\mathrm{pH}$. Epinephrine (E) used in in vitro experiments with blood cells (Suprarenin $1 \mathrm{mg} / \mathrm{mL}$ ) was from Sanofi Aventis, Bridgewater, NJ, USA.

2.2. Blood Sampling. Blood sampling was performed according to the Helsinki declaration considering research on humans, the Oviedo convention on human rights and biomedicine, and the Slovenian medical deontology codex. The study was approved by the Slovenian National Medical Ethics Committee, number 117/02/10. No adverse effects on donors' health due to sampling were observed.

For isolation of EVs, blood was withdrawn from the median cubital vein with 21-gauge needle (MULTI Sample Needle, Nipro Corporation, Tokyo, Japan) using $2.7 \mathrm{~mL}$ vacutubes which contained $270 \mu \mathrm{L}$ of sodium citrate at a concentration $0.109 \mathrm{~mol} / \mathrm{L}$ (Becton Dickinson, New Jersey, USA). Variation in the acquired volumes of blood did not exceed $15 \%$. During the processing, the tubes and blood samples were kept in thermoblocks at $37^{\circ} \mathrm{C}$.

For in vitro experiments with blood and added lidocaine/epinephrine, $8-12 \mathrm{~mL}$ of blood was taken from each of the three authors without a record of the disease (1 male and 2 females, average age 38 years) as described above. For in vitro experiments with plasma and added lidocaine/epinephrine, 8-12 mL of blood was taken from each of the three volunteers without a record of the disease (female, average age 24 years) as described above.

For analysis of the effect of L/E, L, and E on the blood cell membranes, a drop of blood was taken from each of the two volunteers with no record of disease (female, average age 41 years). The drop of blood was taken with a pipette from a small incision on a fingertip. Blood was inserted into an Eppendorf tube previously filled with $1 \mathrm{~mL}$ of citrated phosphate-buffered saline (PBS). The sample was further diluted to obtain a required density of blood cells for observation. The required density rendered a monolayer of

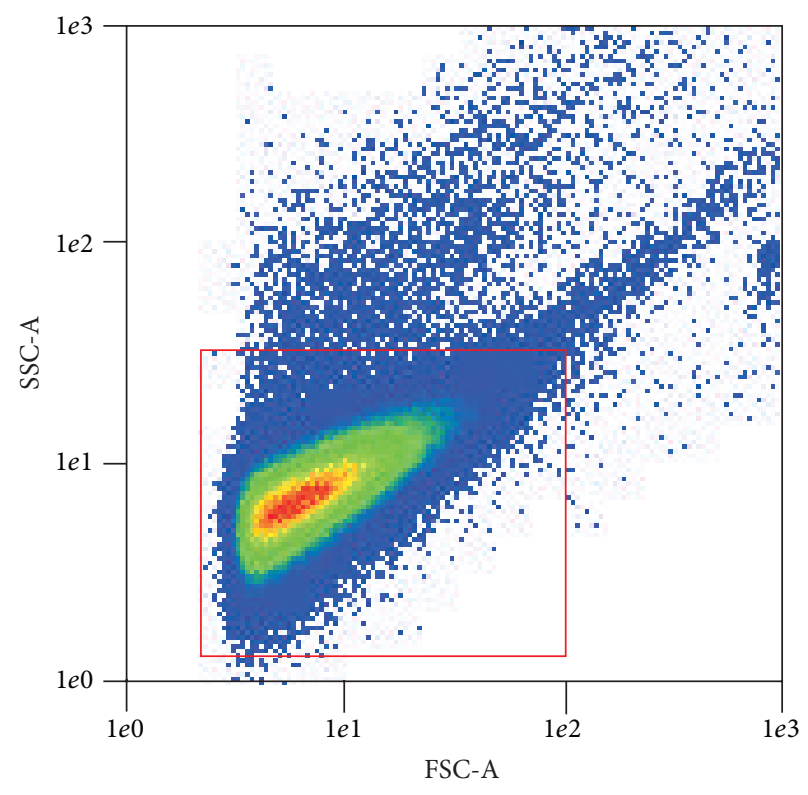

FIGURE 1: Density plot (forward scattered light FSC-A versus side scattered light SSC-A) representing extracellular vesicles (EVs) isolated from a sample of blood incubated with lidocaine/epinephrine (L/E) [39]. The region within the red rectangle estimates events due to EVs.

relatively closely packed cells in an observation frame under the microscope.

2.3. Isolation of EVs from Plasma. Within each sampling session, processing of blood started within 20 minutes from the sampling of the first sample. Blood was centrifuged at $1550 \mathrm{~g}$ and $37^{\circ} \mathrm{C}$ for 20 minutes in a Centric 200/R centrifuge (Domel d.o.o., Železniki, Slovenia). Upper $250 \mu \mathrm{L}$ of plasma was slowly removed and placed in a $1.8 \mathrm{~mL}$ Eppendorf microtube. The samples were then centrifuged at $17570 \mathrm{~g}$ for 5 minutes in a Centric 200/R centrifuge (Domel d.o.o., Železniki, Slovenia), so that EVs were gathered on the bottom of the microtube. The upper $210 \mu \mathrm{L}$ of plasma was discarded and the remaining $40 \mu \mathrm{L}$ of pellet was vortexed at $1200 \mathrm{~g}$ and resuspended in $210 \mu \mathrm{L}$ of PBS ( $\mathrm{pH}=7.4$ ). The samples were centrifuged again at $17570 \mathrm{~g}$ for 5 minutes. $210 \mu \mathrm{L}$ of the supernatant was discarded and $40 \mu \mathrm{L}$ of pellet resuspended in $60 \mu \mathrm{L}$ of citrated phosphate-buffered saline ( $\mathrm{pH}=7.4)$.

2.4. Flow Cytometric Analysis. Concentration of EVs in isolates was determined by MACSQuant Analyzer (http://www .miltenyibiotec.com/en/products-and-services/macs-flowcytometry/flow-cytometers/macsquant-analyzer-10/macsquantanalyzer-10.aspx) (Miltenyi Biotec GmbH, Bergisch-G ladbach, Germany) flow cytometer with $405 \mathrm{~nm}, 488 \mathrm{~nm}$, and $640 \mathrm{~nm}$ air cooled lasers. The MACSQuantify (Miltenyi Biotec GmbH, Bergisch-Gladbach, Germany) software version 2.4 was used for data acquisition and analysis of the results. $25 \mu \mathrm{L}$ of sample was measured. The presence of residual cells and EVs was determined by forward and side scatter parameters. For illustration, Figure 1 shows a flow cytometric scatter diagram (side scatter/forward scatter) of 
EVs isolated from peripheral blood treated with anesthetic L/E.

Typically, the region of events corresponding to EVs (marked by the red rectangle) extends over wide intervals of SSC-A/FSC-A that reflect the detected particle sizes and surface properties. The region contained more than $90 \%$ of events in all density plots.

2.5. Effect of Lidocaine/Epinephrine on EV Concentration in Isolates from Blood. Four $2.7 \mathrm{~mL}$ tubes of blood were taken from each donor and aliquoted into $4 \mathrm{~mL}$ plastic tubes. $500 \mu \mathrm{L}$ of L/E (or PBS for control) was added to each $2 \mathrm{~mL}$ aliquot of blood, gently mixed by turning the tubes upside down, and incubated at $37^{\circ} \mathrm{C}$ for 15 minutes. Blood was centrifuged at $37^{\circ} \mathrm{C}$ and $500 \mathrm{~g}$ for 7 minutes in a Centric 400R centrifuge (Domel d.o.o., Železniki, Slovenia) to divide majority of blood cells from plasma. EVs were then isolated from plasma and counted by the MACSQuant flow cytometer as described above.

2.6. Effect of Lidocaine/Epinephrine on EV Concentration in Isolates from Plasma. Four $2.7 \mathrm{~mL}$ tubes of blood were taken from each donor. Blood was centrifuged at $37^{\circ} \mathrm{C}$ and $500 \mathrm{~g}$ for 7 minutes in a Centric 400R centrifuge (Domel d.o.o., Železniki, Slovenia) to divide majority of blood cells from plasma. Plasma occupying the region about $0.5 \mathrm{~cm}$ above the buffy coat was collected in a plastic container and aliquoted into $1.8 \mathrm{~mL}$ Eppendorf tubes. $50 \mu \mathrm{L} \mathrm{L} / \mathrm{E}$ (or PBS for control) was added to $200 \mu \mathrm{L}$ aliquots of plasma. Samples were gently mixed by slowly turning the tubes upside down and incubated for 15 minutes at $37^{\circ} \mathrm{C}$. During the incubation, the samples were every 5 minutes gently mixed by slowly turning the tubes upside down. EVs were then isolated from plasma and counted by the MACSQuant flow cytometer as described above.

2.7. Effect of Lidocaine/Epinephrine, Lidocaine, or Epinephrine on Erythrocyte Shape. To observe the effect of L/E on erythrocytes, a drop of blood was dissolved in $1 \mathrm{~mL}$ of phosphate buffered saline. $500 \mu \mathrm{L}$ of L/E, L, E, or PBS for control was added to the test samples and observed immediately under the Leitz Aristoplan (Leitz, Wetzlar, Germany) optical microscope equipped by the Watec (Model: 902DM3S), Watec Inc., New York, USA, camera and Pinnacle Studio HD, Version 15.0.0.7593 frame grabber and software, Avid Technology Inc., USA. An observation chamber $1.5 \times 1 \mathrm{~cm}^{2}$ was created on the glass by using silicon grease. $40 \mu \mathrm{L}$ of the test suspension was placed in the observation chamber and closed by the cover glass. Care was taken not to leave voids in the grease boundary in order to prevent evaporation of liquid from the observation chamber. For quantitative analysis of the effect of $\mathrm{L} / \mathrm{E}, \mathrm{L}$, or $\mathrm{E}$ on blood cell membranes, we randomly imaged at least 50 frames over the sample. We avoided regions close to the silicon grease. Two independent experiments using blood of two donors without record of disease were performed for each substance tested.

2.8. Image Analysis of Blood Cell Populations. The image analysis was performed by two authors blinded with respect
TABLE 1: Number of events within the chosen flow cytometer SSCA/FSC-A region corresponding to platelets and EVs (Figure 1) in the isolate from blood treated with $\mathrm{L} / \mathrm{E}$.

\begin{tabular}{lcccc}
\hline & $\begin{array}{c}\text { Subject 1 } \\
(1 / \mu \mathrm{L})\end{array}$ & $\begin{array}{c}\text { Subject 2 } \\
(1 / \mu \mathrm{L})\end{array}$ & $\begin{array}{c}\text { Subject 3 } \\
(1 / \mu \mathrm{L})\end{array}$ & $\begin{array}{c}\text { Average } \\
(1 / \mu \mathrm{L})\end{array}$ \\
\hline $\begin{array}{l}\text { Control } \\
\text { (added PBS) }\end{array}$ & 2602 & 3325 & 2065 & 2664 \\
\hline $\begin{array}{l}\text { Test } \\
(\text { added L/E) }\end{array}$ & 9290 & 9236 & 8670 & 9065 \\
\hline
\end{tabular}

SSC-A/FSC-A: side scattered light/forward scattered light; EVs: extracellular vesicles; PBS: citrated and phosphate-buffered saline; L/E: lidocaine/epinephrine. Blood was donated by three authors with no record of disease and was processed immediately after the addition of $\mathrm{L} / \mathrm{E}$. The region contained more than $90 \%$ of events in all density plots.

TABLE 2: Number of events within the chosen flow cytometer SSCA/FSC-A region corresponding to platelets and EVs (Figure 1) in the isolate from platelet-rich plasma treated with $\mathrm{L} / \mathrm{E}$.

\begin{tabular}{lcccc}
\hline & $\begin{array}{c}\text { Subject 1 } \\
(1 / \mu \mathrm{L})\end{array}$ & $\begin{array}{c}\text { Subject 2 } \\
(1 / \mu \mathrm{L})\end{array}$ & $\begin{array}{c}\text { Subject 3 } \\
(1 / \mu \mathrm{L})\end{array}$ & $\begin{array}{c}\text { Average } \\
(1 / \mu \mathrm{L})\end{array}$ \\
\hline $\begin{array}{l}\text { Control } \\
\text { (added PBS) }\end{array}$ & 1090 & 1500 & 1330 & 1306 \\
\hline $\begin{array}{l}\text { Test } \\
\text { (added L/E) }\end{array}$ & 4820 & 2820 & 3420 & 3687 \\
\hline
\end{tabular}

SSC-A/FSC-A: side scattered light/forward scattered light; EVs: extracellular vesicles; PBS: citrated and phosphate-buffered saline; L/E: lidocaine/epinephrine. Blood was donated by three volunteers with no record of disease. Plasma with added anesthetic L/E was incubated for 15 minutes prior to isolation of EVs. The region contained more than $90 \%$ of events in all density plots.

to each other. For each sample, we created an ensemble of pictures from which we omitted pictures of invalid quality. We counted the number of discocytes, echinocytes, and stomatocytes in each picture. It was assumed that each picture was representative for the sample and that the number of cells of each type fluctuated around the corresponding average value. The average values of the percent of discocytes, echinocytes, and stomatocytes were calculated for each sample. Altogether, more than 6000 cells were included in the analysis of each observer.

2.9. Statistical Analysis. To determine the difference between the populations or samples, the two-tailed paired $t$-test was used. The statistical significance of the $t$-test was given by the probability $p$ and statistical power $(P)$. For calculation, we used Microsoft Excel software (Microsoft Office Excel 2007 SP3) and Power \& Sample Size Calculator.

\section{Results and Discussion}

Results of in vitro experiments where the local anesthetic L/E was added to samples of blood and plasma are presented in Tables 1 and 2, respectively. It can be seen that the concentrations of EVs in the isolate are substantially higher in the respective samples with added anesthetic L/E than in the control samples. The mechanisms underlying this effect are poorly understood, but it was suggested that the isolates 


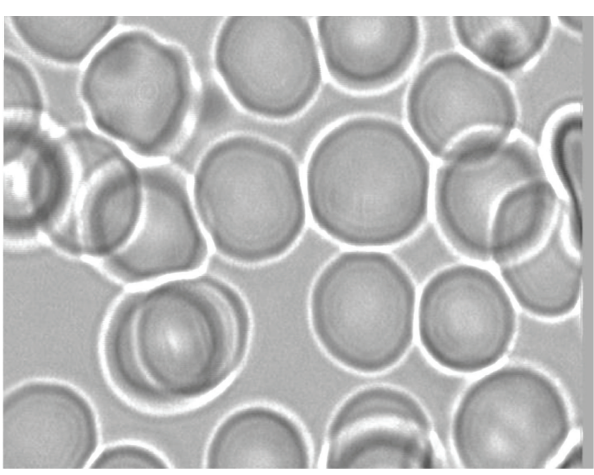

(a)

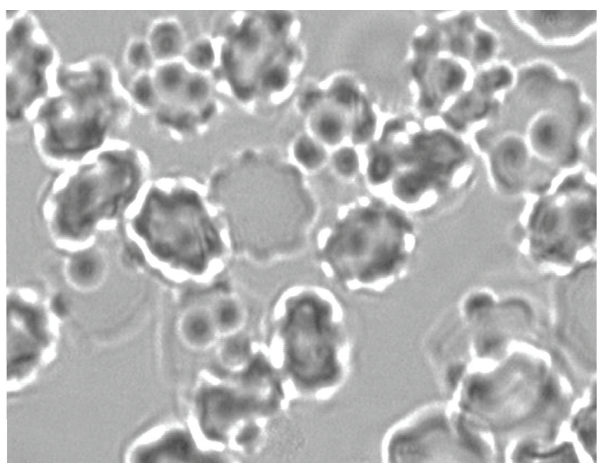

(c)

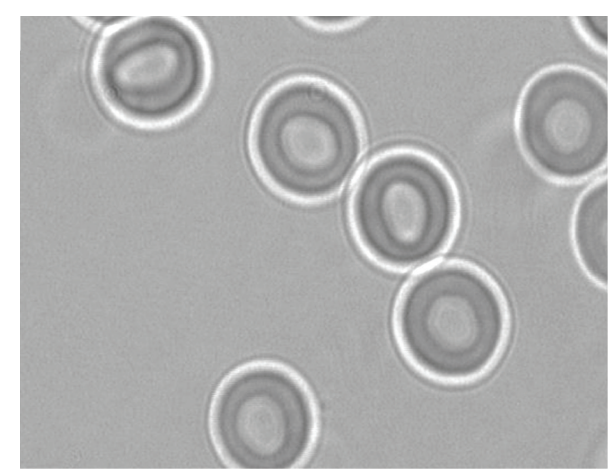

(b)

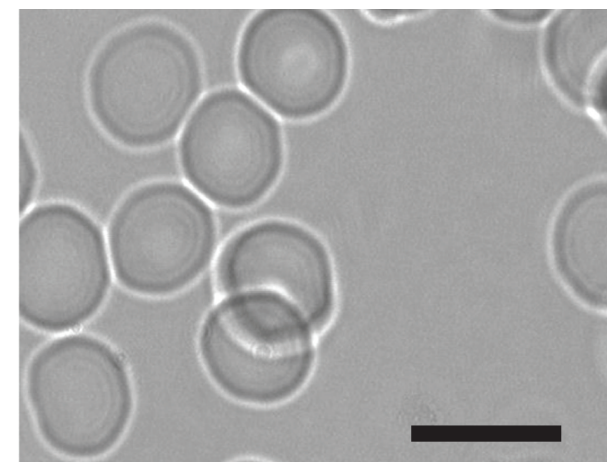

(d)

FIGURE 2: Effect of the local anesthetic lidocaine/epinephrine (L/E) on the erythrocyte shape. (a) Blood dissolved in phosphate buffered saline (PBS), (b) blood dissolved in PBS with added L/E (stomatocytosis), (c) echinocytosis was induced by centrifugation of blood dissolved in PBS, and (d) echinocytes dissolved in PBS transformed into discocytes after the addition of L/E to the suspension. Bar $=10 \mu \mathrm{m}$.

do not contain only EVs that are already present in the sample but predominantly EVs produced during harvesting due to shear forces acting during the centrifugation [40]. The added $\mathrm{L} / \mathrm{E}$ affects rheological properties of the fluid in which cells and fragments are subjected to shear forces in the centrifuge. Also, L/E has an effect on the cell membrane rendering it more prone to vesiculate.

The effect of L/E on erythrocytes can be seen in Figures 2 and 3. Figure 2(a) shows a population of erythrocytes taken from fresh blood: the discocyte shape characteristic for physiological in vitro conditions was observed. By the time the samples with added L/E were mounted to the microscope, the majority of cells attained stomatocytic shapes (Figure 2(b)) which did not change within the observation time (about 1 hour). Centrifugation of blood at $1550 \mathrm{~g}$ for 15 minutes caused echinocytosis (Figure 2(c)). The addition of $\mathrm{L} / \mathrm{E}$ to the previously centrifuged erythrocytes restored discocytic shape in most erythrocytes (Figure 2(d)). Fewer cells were observed in the samples with added anesthetic due to considerable dilution of the samples by the added L/E.

Figure 3 shows a rouleaux formed from L/E treated erythrocytes (Figure 3(b)). Compared to an untreated sample (Figure 3(a)), the packing is less effective. Also, the stomatocyte invaginations attain peculiar nonaxisymmetric shapes (Figures 3(c)-3(i)).

Quantitative analysis of the effect of L/E and its components $\mathrm{L}$ and $\mathrm{E}$ on the erythrocyte shape is presented in
Table 3. Control samples contained mostly discocytes (more than $80 \%$ ), up to about $20 \%$ of echinocytes, and almost no stomatocytes. Due to the effect of the local anesthetic $\mathrm{L} / \mathrm{E}$, the portion of echinocytes dropped to minute values while the portion of stomatocytes considerably increased to $56 \%-90 \%$. The effect of L was even more pronounced; here, almost all erythrocytes (96\%-99\%) attained stomatocytic shape. The effect of E was opposite: discocytes transformed into echinocytes which prevailed (91\%-100\%) while no stomatocytes were observed in the samples treated with E. The differences in the portions of prevailing erythrocyte shapes for all three substances were strongly statistically significant $\left(p<10^{-8}\right)$ with the power at $\alpha=0.05$ equal to 1 .

The direct effect of $\mathrm{L} / \mathrm{E}$ (and its components $\mathrm{L}$ and E) on cell membranes is reflected in the shape of affected erythrocytes which can be observed live under the optical microscope. An erythrocyte has no internal structure and attains a shape corresponding to the properties of its membrane [32, 33].

Relative expansion of the inner membrane with respect to its bilayer couple (Figure 4) was previously proposed as the mechanism underlying shape transformation in erythrocytes [31] and supported by theoretical models. The models have shown that stomatocytic shape has at given membrane area and enclosed volume the lowest difference between the areas of the outer and the inner membrane layer $(\Delta A)$, followed by the discocytic shape [40] and echinocytic shape [41, 42]. If 


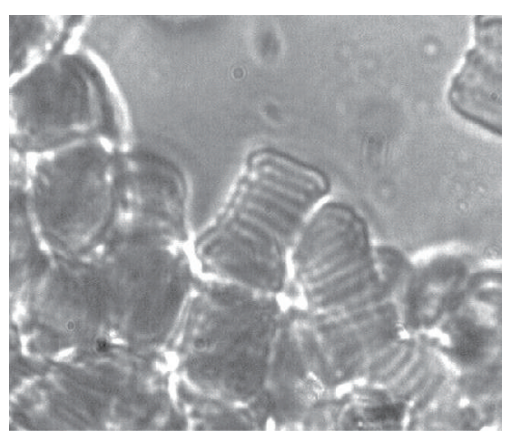

(a)

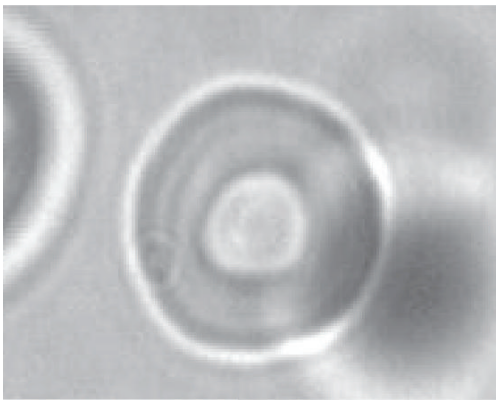

(d)

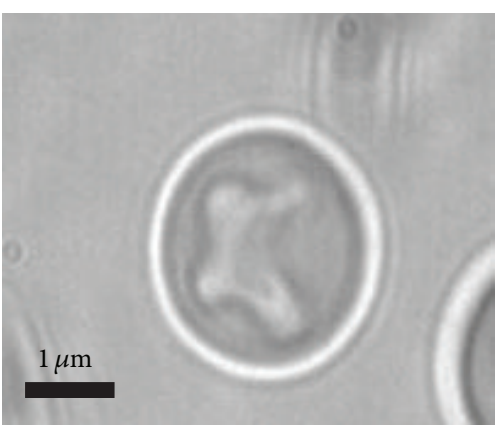

(g)

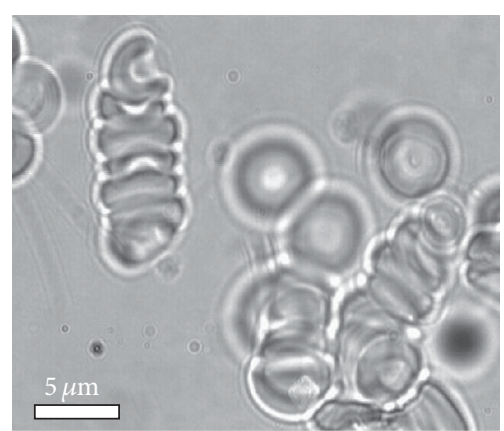

(b)

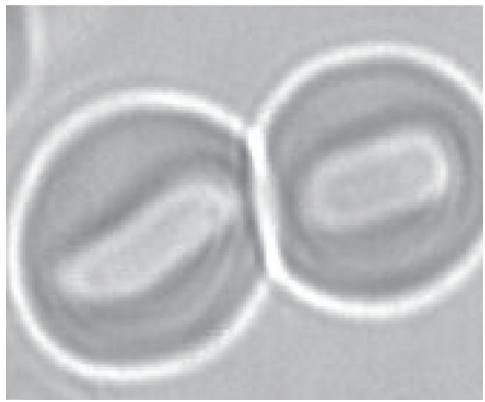

(e)

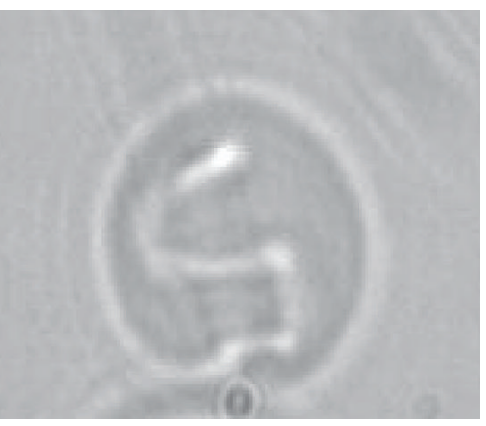

(h)

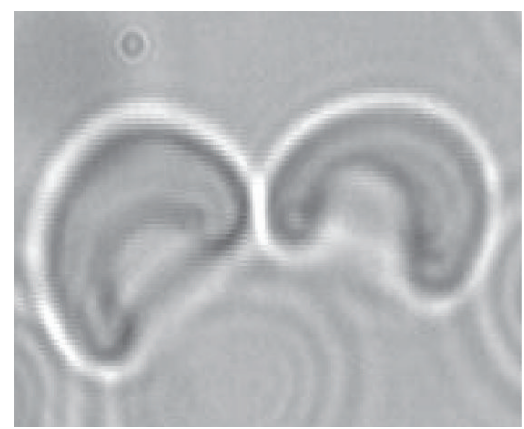

(c)

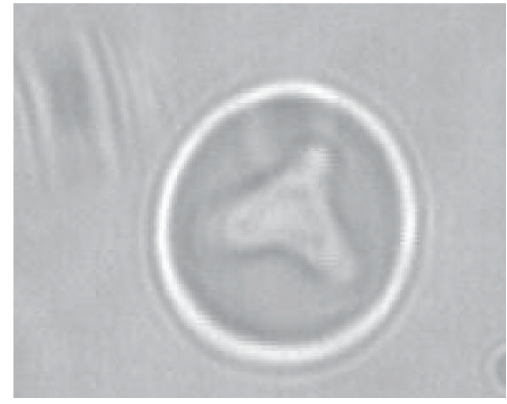

(f)

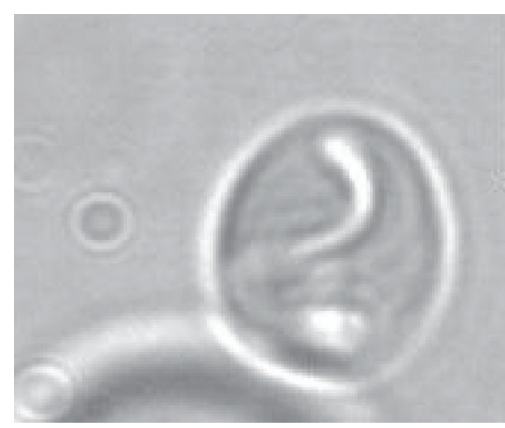

(i)

Figure 3: Effect of the local anesthetic lidocaine/epinephrine (L/E) on the erythrocyte shape. (a) Erythrocyte rouleaux formed in blood plasma, (b) erythrocyte rouleaux formed in blood plasma with added L/E, and (c) side view and (d)-(i) top view of different stomatocyte shapes found in blood dissolved in PBS with added L/E.

the membrane is thin with respect to the inverse curvature in any point at the surface, the area difference of the erythrocyte is given by the geometrical relation

$$
\Delta A=h \int\left(C_{1}+C_{2}\right) \mathrm{d} A,
$$

where $h$ is the effective membrane thickness, $C_{1}$ and $C_{2}$ are the principal curvatures of the membrane at a given point at the membrane surface, and $\mathrm{d} A$ is the area element. The integration is performed over the surface area of the erythrocyte $A$. The average mean curvature of the erythrocyte $\langle H\rangle$ is defined as

$$
\langle H\rangle=\frac{1}{2 A} \int\left(C_{1}+C_{2}\right) \mathrm{d} A .
$$

It follows from (1) and (2) that changes in area difference and average mean curvature are proportional:

$$
\langle H\rangle=\frac{\Delta A}{2 h A} .
$$

By analyzing ensembles of images of diluted blood samples treated with L/E, L, E, and PBS (for control), we found that erythrocytes having on average discocytic shape became on average stomatocytic after the addition of L/E (Table 3, Figures 2(a) and 2(b)), which indicates a decrease of the average mean curvature of the membrane. This effect was confirmed when we observed that adding L/E to echinocytes (induced by centrifugation of blood) yielded discocytes (Figures 2(c) and $2(\mathrm{~d})$ ). E had an opposite effect on the membrane; erythrocytes having on average discocytic shape became on average echinocytic after the addition of $\mathrm{E}$ (Table 3 ). Therefore the stomatocytic effect of the combined compound 


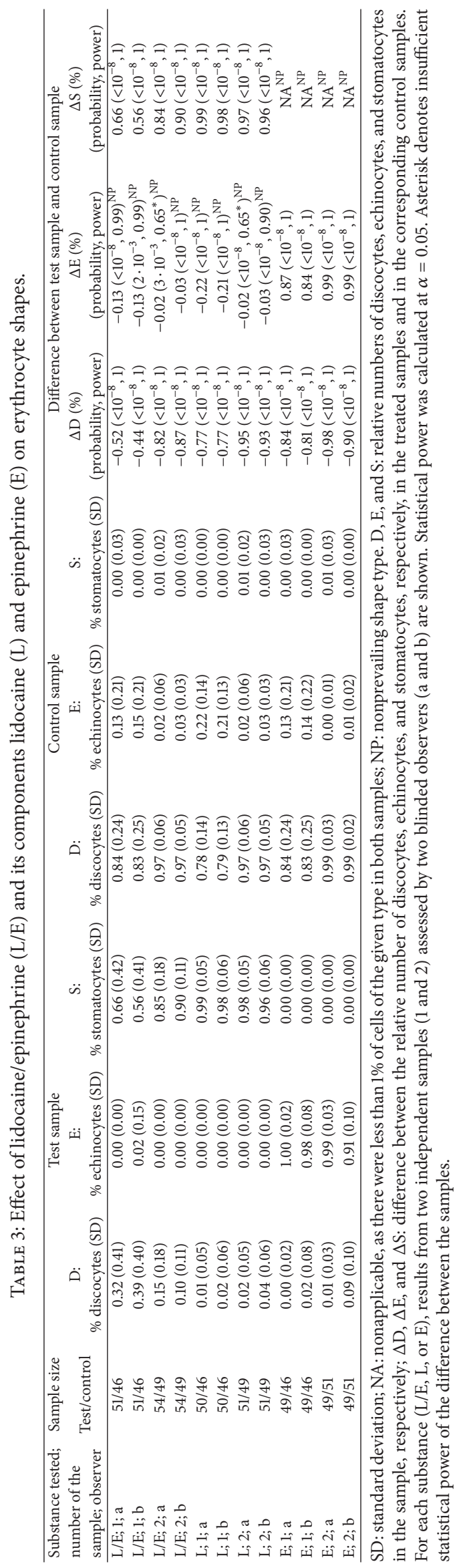



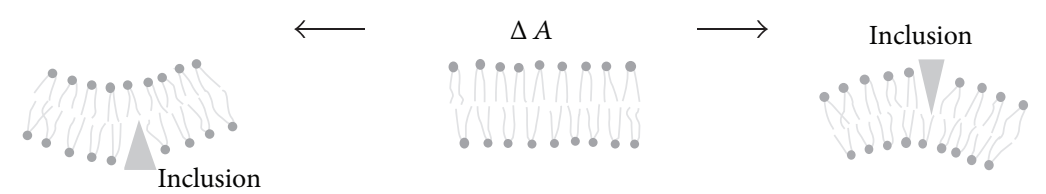

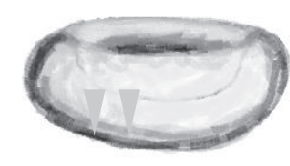

(b)

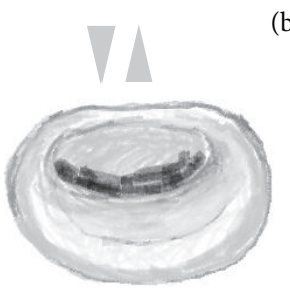

(d)

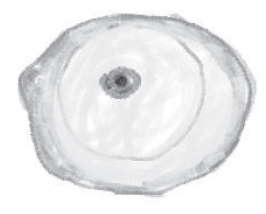

(f)

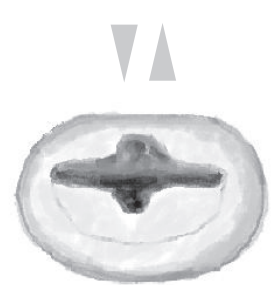

(e)

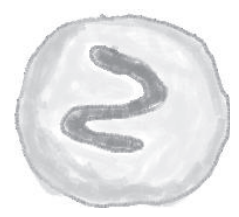

(g)

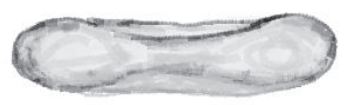

(a)

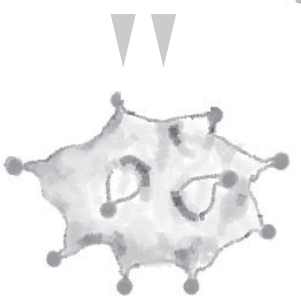

(h)

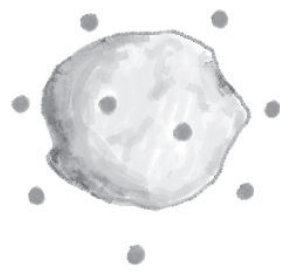

(j)

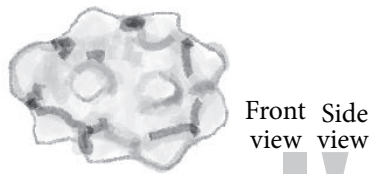

(c)

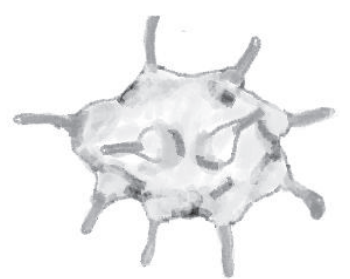

(i)

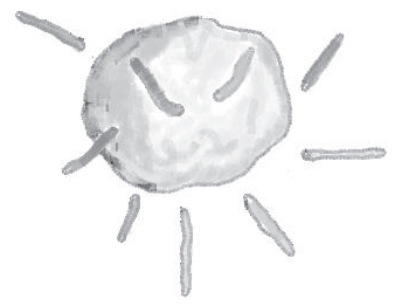

(k)

FIGURE 4: Suggested mechanism of erythrocyte shape change due to continuous interaction of exogenous substance with the membrane. Initially discocytic shape (a) transforms due to inclusions seeded by exogenous substance. If the inclusion resides in the outer layer, the area of the outer layer expands relatively to the area of the inner layer (the difference between the areas of the outer and the inner layer $\Delta A$ increases) and the membrane wrinkles outwards to attain echinocytic shape (c). If the inclusion intercalates into the inner layer, the area of the inner layer expands relatively to the area of the outer layer ( $\triangle A$ decreases) and the membrane bends inwards to attain stomatocytic shape (b). The process is promoted by increasing the number of inclusions while the erythrocyte shape transformation depends on the shape of the inclusion. If the inclusion is isotropic (axisymmetric with respect to the axis perpendicular to the membrane surface) and favors strongly curved membrane regions, membrane buds that form on the tips of the echinocyte spicules have spherical shape (h) while the invagination of the stomatocyte is axisymmetric with respect to the geometrical axis of the cell (d). If the inclusion is anisotropic (nonaxisymmetric with respect to the axis perpendicular to the membrane surface, so that its front view differs from its side view) and favors strongly curved cylindrical membrane regions, the membrane buds that form on the tips of the echinocyte spicules have tubular shape (i), while the invagination of the stomatocyte attains a peculiar pocket-like shape (e). The process of shape transformation is reflected also in the shape of the pinched-off EVs which may be spherical (j) or tubular (k) or in the shape of erythrocyte inner structures hinted by the shape of the closure at the cell surface (f, $g$ ), depending on the shape of the inclusions and their preference for the outer/inner membrane layer.

$\mathrm{L} / \mathrm{E}$ was somewhat less pronounced than the effect of $\mathrm{L}$ alone (Table 3), although the effect of L in L/E strongly prevailed.

Our experiments demonstrated that erythrocytes on average changed shape after the addition of $\mathrm{L} / \mathrm{E}$, $\mathrm{L}$, or $\mathrm{E}$ (Table 3 ) in such way that the average mean curvature was changed. The change of the average mean curvature was unambiguous, although osmotic effects and changes of the membrane elastic properties could also have taken place. It was therefore interpreted that the added substances interacted with erythrocyte membrane and affected its average mean curvature. However, this mechanism alone cannot explain peculiar shapes of invaginations observed in Figures 3(e)-3(i). A large portion of stomatocytes in the samples treated with $\mathrm{L} / \mathrm{E}$ or $\mathrm{L}$ had invaginations with complex cross sections (Figures 3(e)-3(g)) that developed into flattened pocket-like structures (Figures $3(\mathrm{~h})$ and 3(i)). Based on previous evidence with similar shape transformation [38], formation of anisotropic inclusions (inclusions lacking the symmetry with respect to the axis perpendicular to the membrane surface: see the scheme of the inclusions pertaining to shapes (e) and (g) in Figure 4) takes place during the interaction of $\mathrm{L}$ with the membrane. These inclusions due to their shape energetically prefer regions with different principal curvatures of the membrane at bulby ends of the flattened invaginations (see also [38]).

Shape transformation was in this work observed and reported only in erythrocytes, but the mechanism based on formation of inclusions and their accumulation in particular membrane regions that leads to formation of extracellular vesicles in general and relevant for all cell types, although 
similarities of the changes of the global shapes of other cells cannot be expected due to different cytoskeletal and other constraints.

As regards $\mathrm{L} / \mathrm{E}$, its effect on the vesiculability of platelets is of utmost importance as nanovesiculation of platelets is considered to be involved in thromboembolic events. Furthermore, platelet-derived EVs were found to prevail in isolates from blood [20]. We have observed increased vesiculability of membranes in $\mathrm{L} / \mathrm{E}$ treated samples of and in platelet-rich plasma where the majority of erythrocytes were prior to addition of L/E to the sample eliminated by "slow" centrifugation. We interpret that more EVs were found in isolates from $\mathrm{L} / \mathrm{E}$ treated samples than in isolates from control samples because $\mathrm{L} / \mathrm{E}$ treated membranes were more prone to vesiculate due to formation of L/E-induced inclusions which changed local membrane curvature and elastic properties of the membrane. However, it should be borne in mind that the content of the isolate is subject to isolation procedure that includes centrifugation of samples with accelerations up to 20000 times the gravity constant $g$. Addition of L/E to the sample also changes the composition of the liquid in which the cells move during the centrifugation, most importantly its viscosity, and therefore affects the shear forces on cells and their fragments. As it was found that a large pool of EVs in blood isolates is produced during the isolation process with shear forces in the centrifuge playing an important role [43], the origin of the EVs found in the isolates cannot be revealed solely from their concentration. Further, mechanisms of EV fusion with cells and other EVs during the isolation are largely unexplored but are likely to take place since fusion of EVs with cells has been observed in cell cultures [44]. It can however be extrapolated that increased vesiculability of blood cells (especially platelets) due to $\mathrm{L} / \mathrm{E}$ will be revealed in the in vitro conditions and that the observed artefacts are clinically relevant.

It can be seen in Figure 3(b) that in the L/E treated samples the erythrocytes (stomatocytes) are not optimally packed into the rouleaux (as is the case in untreated samples). If such structures took shape in vivo, they could increase the risk for occlusions in the vessels. Further, it was previously found that $\mathrm{E}$ has marked effect on platelets. Microaggregation of platelets, recruitment into circulation, E binding, and change of platelet volume have been reported in vivo [45] while platelet activation was observed in connection to shear forces when $\mathrm{E}$ was added to blood in vitro [46].

Blood cells are a convenient system for the study of the effect of various substances on biological membranes as they are relatively easily accessible and can be observed in vitro in an environment that is similar to in vivo situation. This enables direct envisaging of relevant features. The development of ethically unbiased methods is of importance in design of paradigms for testing substances impacts and toxicity [47-49], for which there is an increasing need in technology development as well as in diagnostics.

\section{Conclusions}

$\mathrm{L} / \mathrm{E}$ interacts with cell membranes and increases vesiculability of blood cells in vitro, presumably due to induction of inclusions in the membrane that favour strongly curved membrane regions. Accumulation of these inclusions on membrane buds drives the membrane to form and shed nanovesicles. Ensembles of blood cells are a convenient system for the study of the effect of different added substances on properties of biological membranes.

\section{Conflict of Interests}

The authors declare that there is no conflict of interests regarding the publication of this paper.

\section{Acknowledgments}

The authors are thankful for support from ARRS Grants J16728, J3-5499, and P3-0388.

\section{References}

[1] R. P. Richter, R. Bérat, and A. R. Brisson, "Formation of solidsupported lipid bilayers: an integrated view," Langmuir, vol. 22, no. 8, pp. 3497-3505, 2006.

[2] C. Tetta, E. Ghigo, L. Silengo, M. C. Deregibus, and G. Camussi, "Extracellular vesicles as an emerging mechanism of cell-to-cell communication," Endocrine, vol. 44, no. 1, pp. 11-19, 2013.

[3] J. Rak, "Extracellular vesicles-biomarkers and effectors of the cellular interactome in cancer," Frontiers in Pharmacology, vol. 4, article 21, 2013.

[4] K. Schara, V. Janša, V. Šuštar et al., "Mechanisms for the formation of membranous nanostructures in cell-to-cell communication," Cellular and Molecular Biology Letters, vol. 14, no. 4, pp. 636-656, 2009.

[5] G. Müller, "Microvesicles/exosomes as potential novel biomarkers of metabolic diseases," Diabetes, Metabolic Syndrome and Obesity: Targets and Therapy, vol. 5, pp. 247-282, 2012.

[6] A. Mrvar-Brečko, V. Šuštar, V. Janša et al., "Isolated microvesicles from peripheral blood and body fluids as observed by scanning electron microscope," Blood Cells, Molecules, and Diseases, vol. 44, no. 4, pp. 307-312, 2010.

[7] S. El Andaloussi, I. Mäger, X. O. Breakefield, and M. J. A. Wood, "Extracellular vesicles: biology and emerging therapeutic opportunities," Nature Reviews Drug Discovery, vol. 12, no. 5, pp. 347-357, 2013.

[8] Y. Lee, S. El Andaloussi, and M. J. A. Wood, "Exosomes and microvesicles: extracellular vesicles for genetic information transfer and gene therapy," Human Molecular Genetics, vol. 21, no. 1, pp. R125-R134, 2012.

[9] K. M. Danielson and S. Das, "Extracellular vesicles in heart disease: excitement for the future," Exosomes \& Microvesicles, vol. 2, article 1, 2014.

[10] V. Kralj-Iglič, "Stability of membranous nanostructures: a possible key mechanism in cancer progression," International Journal of Nanomedicine, vol. 7, pp. 3579-3596, 2012.

[11] M. Lokar, D. Kabaso, N. Resnik et al., "The role of cholesterolsphingomyelin membrane nanodomains in the stability of intercellular membrane nanotubes," International Journal of Nanomedicine, vol. 7, pp. 1891-1902, 2012.

[12] T. J. Greenwalt, "The how and why of exocytic vesicles," Transfusion, vol. 46, no. 1, pp. 143-152, 2006.

[13] L. Mrówczyńska, U. Salzer, A. Iglič, and H. Hägerstrand, "Curvature factor and membrane solubilization, with particular 
reference to membrane rafts," Cell Biology International, vol. 35, no. 10, pp. 991-995, 2011.

[14] A. S. Leroyer, F. Anfosso, R. Lacroix et al., "Endothelialderived microparticles: biological conveyors at the crossroad of inflammation, thrombosis and angiogenesis," Thrombosis and Haemostasis, vol. 104, no. 3, pp. 456-463, 2010.

[15] G. Camussi, M. C. Deregibus, S. Bruno, V. Cantaluppi, and L. Biancone, "Exosomes/microvesicles as a mechanism of cell-tocell communication," Kidney International, vol. 78, no. 9, pp. 838-848, 2010.

[16] G. Camussi, M. C. Deregibus, S. Bruno, C. Grange, V. Fonsato, and C. Tetta, "Exosome/microvesicle-mediated epigenetic reprogramming of cells," American Journal of Cancer Research, vol. 1, no. 1, pp. 98-110, 2011.

[17] S. Robert, R. Lacroix, P. Poncelet et al., "High-sensitivity flow cytometry provides access to standardized measurement of small-size microparticles-brief report," Arteriosclerosis, Thrombosis, and Vascular Biology, vol. 32, no. 4, pp. 1054-1058, 2012.

[18] D. P. Romancino, G. Paterniti, Y. Campos et al., "Identification and characterization of the nano-sized vesicles released by muscle cells," FEBS Letters, vol. 587, no. 9, pp. 1379-1384, 2013.

[19] Y. Yuana, A. Sturk, and R. Nieuwland, "Extracellular vesicles in physiological and pathological conditions," Blood Reviews, vol. 27, no. 1, pp. 31-39, 2013.

[20] M. Diamant, R. Nieuwland, R. F. Pablo, A. Sturk, J. W. A. Smit, and J. K. Radder, "Elevated numbers of tissue-factor exposing microparticles correlate with components of the metabolic syndrome in uncomplicated type 2 diabetes mellitus," Circulation, vol. 106, no. 19, pp. 2442-2447, 2002.

[21] R. Janša, V. Šuštar, M. Frank et al., "Number of microvesicles in peripheral blood and ability of plasma to induce adhesion between phospholipid membranes in 19 patients with gastrointestinal diseases," Blood Cells, Molecules, and Diseases, vol. 41, no. 1, pp. 124-132, 2008.

[22] V. Suštar, A. Bedina-Zavec, R. Stukelj et al., "Post-prandial rise of microvesicles in peripheral blood of healthy human donors," Lipids in health and disease, vol. 10, article 47, 2011.

[23] R. Štukelj, V. Suštar, A. Mrvar-Brečko et al., "Suppression of membrane vesiculation as anticoagulant and anti-metastatic mechanism. Role of stability of narrow necks," General Physiology and Biophysics, vol. 32, no. 1, pp. 33-45, 2013.

[24] I. Müller, A. Klocke, M. Alex et al., "Intravascular tissue factor initiates coagulation via circulating microvesicles and platelets.", The FASEB Journal, vol. 17, no. 3, pp. 476-478, 2003.

[25] U. Rauch and S. Antoniak, "Tissue factor-positive microparticles in blood associated with coagulopathy in cancer," Thrombosis and Haemostasis, vol. 97, no. 1, pp. 9-10, 2007.

[26] R. J. Berckmans, R. Nieuwland, A. N. Böing, F. P. H. T. M. Romijn, C. E. Hack, and A. Sturk, "Cell-derived microparticles circulate in healthy humans and support low grade thrombin generation," Thrombosis and Haemostasis, vol. 85, no. 4, pp. 639646, 2001.

[27] M. Ikeda, S.-I. Iwamoto, H. Imamura, H. Furukawa, and T. Kawasaki, "Increased platelet aggregation and production of platelet-derived microparticles after surgery for upper gastrointestinal malignancy," Journal of Surgical Research, vol. 115, no. 2, pp. 174-183, 2003.

[28] S. Nomura, A. Imamura, M. Okuno et al., "Platelet-derived microparticles in patients with arteriosclerosis obliterans: enhancement of high shear-induced microparticle generation by cytokines," Thrombosis Research, vol. 98, no. 4, pp. 257-268, 2000 .
[29] M. A. Bohensky, R. deSteiger, C. Kondogiannis et al., "Adverse outcomes associated with elective knee arthroscopy: a population-based cohort study," Arthroscopy, vol. 29, pp. 716-725, 2013.

[30] K. T. Delis, N. Hunt, R. K. Strachan, and A. N. Nicolaides, "Incidence, natural history and risk factors of deep vein thrombosis in elective knee arthroscopy," Thrombosis and Haemostasis, vol. 86, no. 3, pp. 817-821, 2001.

[31] M. P. Sheetz and S. J. Singer, "Biological membranes as bilayer couples. A molecular mechanism of drug erythrocyte interactions," Proceedings of the National Academy of Sciences of the United States of America, vol. 71, no. 11, pp. 4457-4461, 1974.

[32] A. Iglič, "A possible mechanism determining the stability of spiculated red blood cells," Journal of Biomechanics, vol. 30, no. 1, pp. 35-40, 1997.

[33] H. Hägerstrand, V. Kralj-Igličc, M. Fošnarič et al., "Endovesicle formation and membrane perturbation induced by polyoxyethyleneglycolalkylethers in human erythrocytes," Biochimica et Biophysica Acta-Biomembranes, vol. 1665, no. 1-2, pp. 191200, 2004.

[34] A. Wróbel, M. Bobrowska-Hägerstrand, C. Lindqvist, and H. Hägerstrand, "Monitoring of membrane phospholipid scrambling in human erythrocytes and K562 cells with FM1-43a comparison with annexin V-FITC," Cellular and Molecular Biology Letters, vol. 19, no. 2, pp. 262-276, 2014.

[35] A. Wróbel, "Effects of charged amphiphiles in depolarising solutions on potassium efflux and the osmotic fragility of human erythrocytes," Bioelectrochemistry, vol. 73, no. 2, pp. 117$122,2008$.

[36] M. Suwalsky, J. Belmar, F. Villena, M. J. Gallardo, M. JemiolaRzeminska, and K. Strzalka, "Acetylsalicylic acid (aspirin) and salicylic acid interaction with the human erythrocyte membrane bilayer induce in vitro changes in the morphology of erythrocytes," Archives of Biochemistry and Biophysics, vol. 539, no. 1, pp. 9-19, 2013.

[37] M. Šimundić, B. Drašler, V. Šuštar et al., "Effect of engineered $\mathrm{TiO}_{2}$ and $\mathrm{ZnO}$ nanoparticles on erythrocytes, platelet-rich plasma and giant unilamelar phospholipid vesicles," BMC Veterinary Research, vol. 9, article 7, 2013.

[38] M. Bobrowska-Hägerstrand, V. Kralj-Iglič, A. Iglič, K. Bialkowska, B. Isomaa, and H. Hägerstrand, "Torocyte membrane endovesicles induced by octaethyleneglycol dodecylether in human erythrocytes," Biophysical Journal, vol. 77, no. 6, pp. 3356-3362, 1999.

[39] V. Suštar, A. Bedina-Zavec, R. Stukelj et al., "Post-prandial rise of microvesicles in peripheral blood of healthy human donors," Lipids in Health and Disease, vol. 10, article 47, 2011.

[40] U. Seifert and R. Lipowsky, "Morphology of vesicles," in Handbook of Biological Physics, R. Lipowsky and E. Sackmann, Eds., vol. 1, p. 421, 1995.

[41] G. H. W. Lim, M. Wortis, and R. Mukhopadhyay, "Stomatocytediscocyte-echinocyte sequence of the human red blood cell: evidence for the bilayer-couple hypothesis from membrane mechanics," Proceedings of the National Academy of Sciences of the United States of America, vol. 99, no. 26, pp. 16766-16769, 2002.

[42] A. Iglič, V. Kralj-Iglič, and H. Hägerstrand, "Amphiphile induced echinocyte-spheroechinoeyte transformation of red blood cell shape," European Biophysics Journal, vol. 27, no. 4, pp. 335-339, 1998.

[43] V. Šuštar, A. Bedina-Zavec, R. Štukelj et al., "Nanoparticles isolated from blood: a reflection of vesiculability of blood 
cells during the isolation process," International Journal of Nanomedicine, vol. 6, pp. 2737-2748, 2011.

[44] E. Ogorevc, S. Hudoklin, P. Veranič, and V. Kralj-Iglič, "Extracellular vesicle-mediated transfer of membranous components from the highly malignant T24 urinary carcinoma cell line to the non-malignant RT4 urinary papilloma cell line," Protoplasma, vol. 251, no. 3, pp. 699-702, 2014.

[45] S. E. Kjeldsen, A. B. Weder, B. Egan, R. Neubig, A. J. Zweifler, and S. Julius, "Effect of circulating epinephrine on platelet function and hematocrit," Hypertension, vol. 25, no. 5, pp. 10961105, 1995.

[46] S. Goto, S. Handa, E. Takahashi, S. Abe, M. Handa, and Y. Ikeda, "Synergistic effect of epinephrine and shearing on platelet activation," Thrombosis Research, vol. 84, no. 5, pp. 351-359, 1996.

[47] T. Hartung, "Toxicology for the twenty-first century," Nature, vol. 460, no. 7252, pp. 208-212, 2009.

[48] L. M. Schechtman, "Implementation of the 3Rs (refinement, reduction, and replacement): validation and regulatory acceptance considerations for alternative toxicological test methods," ILAR Journal, vol. 43, pp. S85-S94, 2002.

[49] N. Hasiwa, M. Daneshian, P. Bruegger et al., "Evidence for the detection of non-endotoxin pyrogens by the whole blood monocyte activation test," ALTEX, vol. 30, pp. 169-208, 2013. 

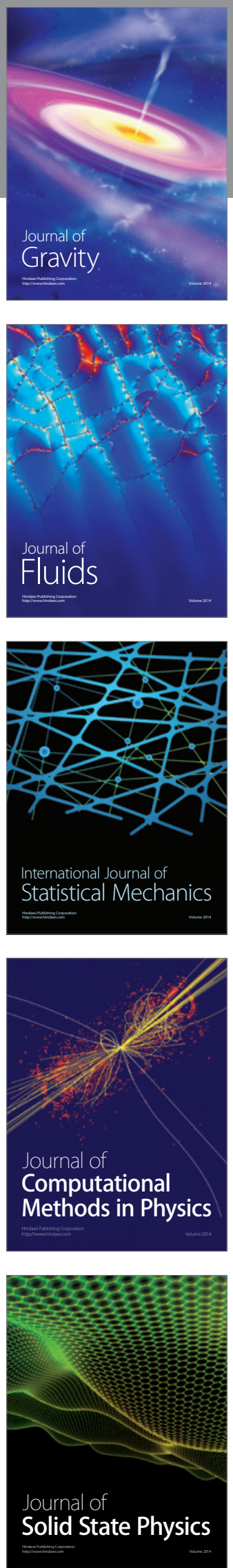

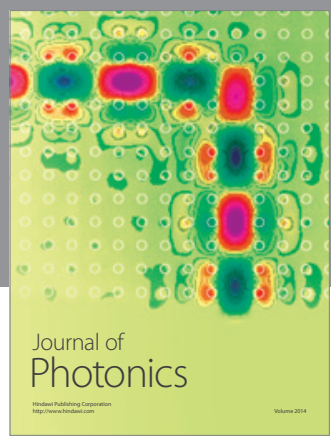

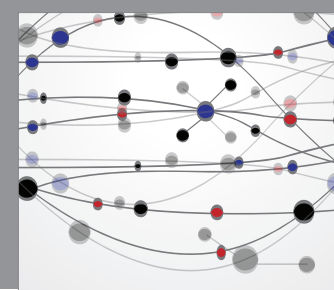

The Scientific World Journal

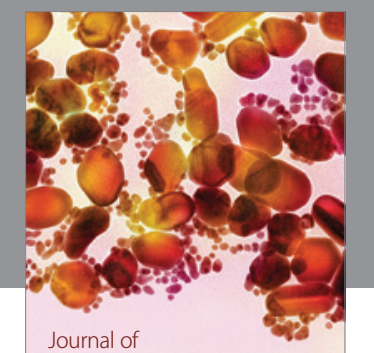

Soft Matter
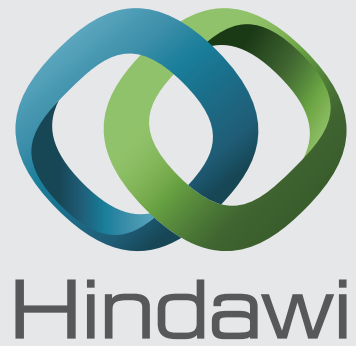

Submit your manuscripts at

http://www.hindawi.com
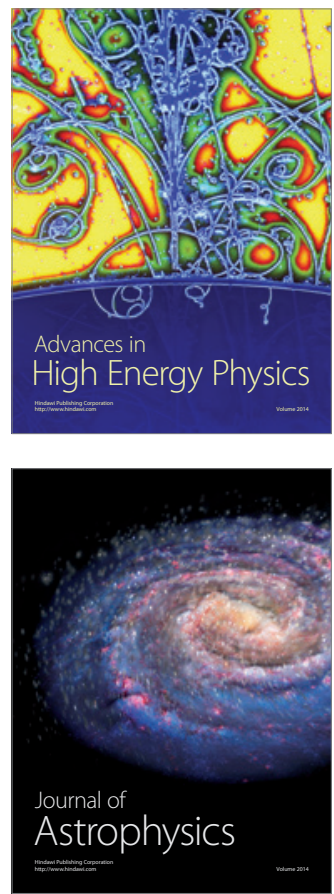
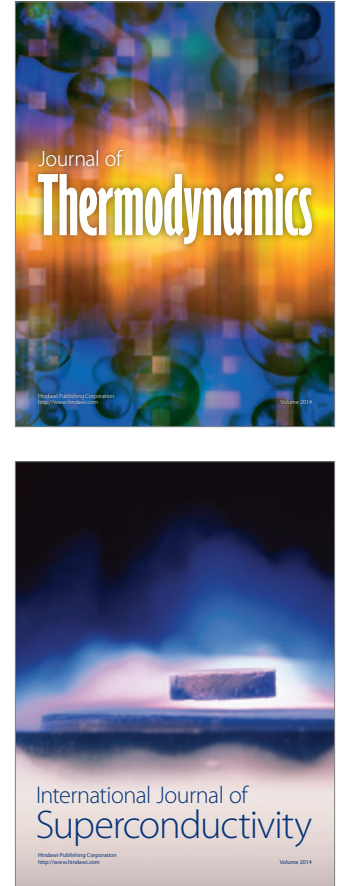
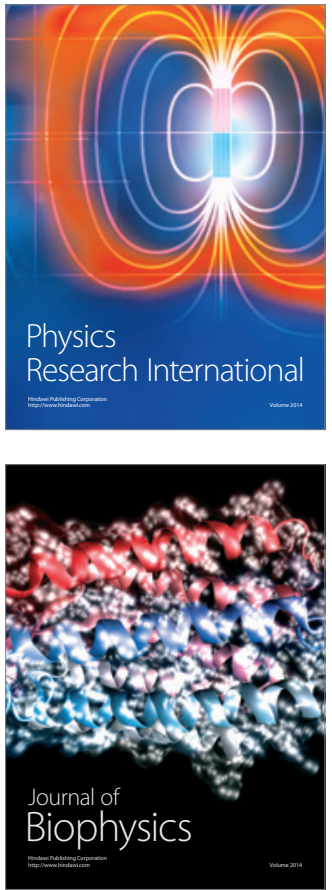
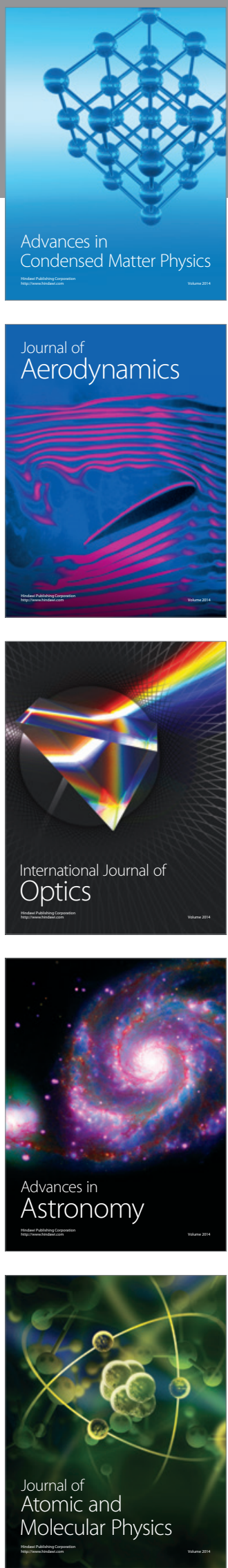